ARTICLE

Received 28 Mar 2014 | Accepted 19 Jun 2014 | Published 17 Jul $2014 \quad$ DOI: 10.1038/ncomms5453

\title{
Excitonic quasiparticles in a spin-orbit Mott insulator
}

Jungho Kim¹, M. Daghofer², A.H. Said', T. Gog ${ }^{1}$, J. van den Brink², G. Khaliullin ${ }^{3}$ \& B.J. Kim³ ${ }^{3,4}$

In condensed matter systems, out of a large number of interacting degrees of freedom emerge weakly coupled quasiparticles (QPs), in terms of which most physical properties are described. The lack of identification of such QPs is a major barrier for understanding myriad exotic properties of correlated electrons, such as unconventional superconductivity and non-Fermi liquid behaviours. Here we report the observation of a composite particle in a quasi-two-dimensional spin-1/2 antiferromagnet $\mathrm{Sr}_{2} \mathrm{IrO}_{4}$-an exciton dressed with magnons - that propagates with the canonical characteristics of a QP: a finite QP residue and a lifetime longer than the hopping time scale. The dynamics of this charge-neutral excitation mirrors the fundamental process of the analogous one-hole propagation in the background of spins-1/2, and reveals the same intrinsic dynamics that is obscured for a single, charged-hole doped into two-dimensional cuprates.

\footnotetext{
${ }^{1}$ Advanced Photon Source, Argonne National Laboratory, Argonne, Illinois 60439, USA. ${ }^{2}$ Institute for Theoretical Solid State Physics, IFW Dresden, Helmholtzstrasse 20, 01069 Dresden, Germany. ${ }^{3}$ Max Planck Institute for Solid State Research, Heisenbergstraße 1, D-70569 Stuttgart, Germany.

${ }^{4}$ Materials Science Division, Argonne National Laboratory, Argonne, Illinois 60439, USA. Correspondence and requests for materials should be addressed to B.J.K. (email: bjkim@fkf.mpg.de).
} 
T he dynamics of a single hole doped into a low-dimensional quantum antiferromagnet (AF) constitutes one of the classic unsolved problems, which has drawn much interest in the context of cuprate high-temperature superconductors ${ }^{1-3}$. In one picture, the single hole forms a coherent QP propagating in a medium of quantum-fluctuating spins, which strongly renormalizes the dispersion relations and the mutual interactions of the holes at finite doping. This spin-polaron picture is supported by numerical approaches, such as quantum Monte Carlo ${ }^{4}$, exact diagonalization ${ }^{5}$ and self-consistent Born approximation $(\mathrm{SCBA})^{2}$. In a contrasting picture, the notion of $\mathrm{QP}$ can break down due to various mechanism such as orthogonality catastrophe ${ }^{6}$, spin-charge separation ${ }^{7}$ and/or localization effects ${ }^{8}$, reflecting the unconventional nature of two-dimensional (2D), strongly interacting fermions. To date, no evidence for a QP has been found in experiments ${ }^{9-12}$ that probe the dynamics of a single hole doped into a $2 \mathrm{D}$ spin-1/2 quantum AFs.

Motivated by the recent discovery of a new pseudospin-1/2 Heisenberg AF on a square lattice, $\mathrm{Sr}_{2} \mathrm{IrO}_{4}$ (refs 13-18), we demonstrate a novel experimental approach using resonant inelastic X-ray scattering (RIXS), which is a rapidly evolving tool especially well suited for such $5 d$ transition-metal oxides, to address this longstanding problem. On the basis of its remarkable similarity to superconducting cuprates in structural ${ }^{18}$, electronic $^{13}$ and magnetic aspects ${ }^{14-16}$, superconductivity has been predicted in $\mathrm{Sr}_{2} \mathrm{IrO}_{4}$ (ref. 19). Although the electron correlation strength in $\mathrm{Sr}_{2} \mathrm{IrO}_{4}$ has been under much debate ${ }^{20-24}$, questioning the validity of classifying this compound as a Mott insulator, $\mathrm{Sr}_{2} \mathrm{IrO}_{4}$ shares the same phenomenology that the hole spectral function as measured by angle-resolved photoemission spectroscopy (ARPES) lacks a legitimate $\mathrm{QP}^{13}$. This similarity suggests a common origin for the absence of a well-defined QP in the ARPES spectra for two distinct but similar classes of materials. As we shall see, however, $\mathrm{Sr}_{2} \mathrm{IrO}_{4}$ supports a well-defined QP in

\section{a}

Hole propagation

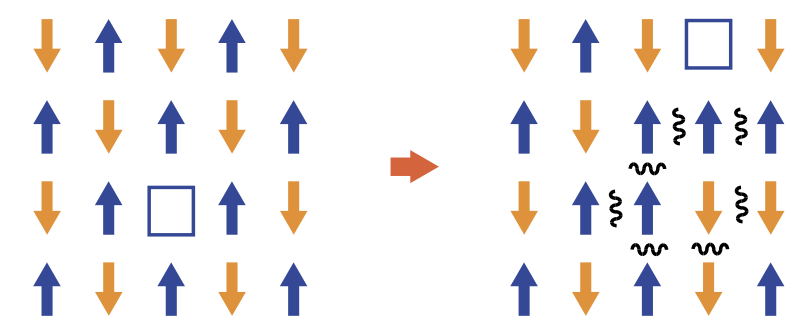

b Exciton propagation

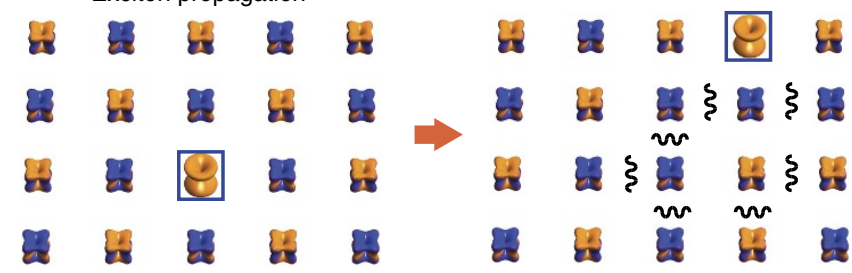

Figure 1 | Hole versus exciton propagation in an antiferromagnetic background. (a) A moving hole (blue square) leaves a string of excited spins behind; that is, every hopping motion of the hole generates a flipping of a spin relative to the ground state configuration. Black wavy lines indicate pairs of 'misaligned' spins. (b) An analogous exciton hopping. While the nature of the spin-orbit exciton (charge-neutral object) and the hole (charged particle) is quite different, they share in common that their hopping creates a string of flipped spins (or isospins in the case of exciton). See Fig. 2 for the description of the isospins and the spin-orbit excitons. another excitation channel, which RIXS is sensitive to and reflects the same dynamics as that of a hole, offering a novel route to studying elementary excitations in correlated oxides.

In an earlier RIXS study of $\mathrm{Sr}_{2} \mathrm{IrO}_{4}$, excitations of a hole across the spin-orbit coupling split levels (from the $j=1 / 2$ manifold to the $j=3 / 2$ manifold) have been identified and it has been shown that their dispersions can be understood in close analogy to the single-hole problem ${ }^{16}$. Figure 1 depicts the hole-versus-exciton analogy: a 'foreign' object injected into a quantum Heisenberg $\mathrm{AF}$, be it a hole or an exciton, creates a string of flipped spins along its hopping path. This analogy, in principle, suggests that the dynamics of a particle moving in a magnetic medium can also be studied using the exciton. However, the energy resolution of RIXS used in the earlier study $(\approx 130 \mathrm{meV})$ was insufficient to resolve the dispersion and the intrinsic linewidth of the twoexciton modes associated with the two pairs of Kramers doublets in the $j=3 / 2$ manifold (Fig. 2a). Here we exploit the different orbital symmetries of the two-exciton modes to selectively probe each mode, and with the much improved energy resolution $(\approx 30 \mathrm{meV})$ offered by RIXS after recent developments, reveal their full dynamics.

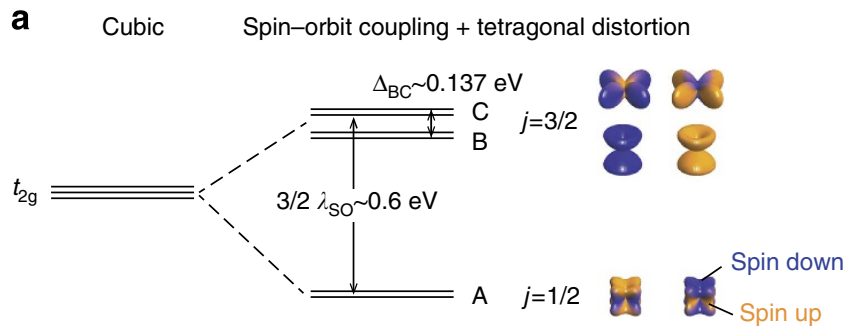

b

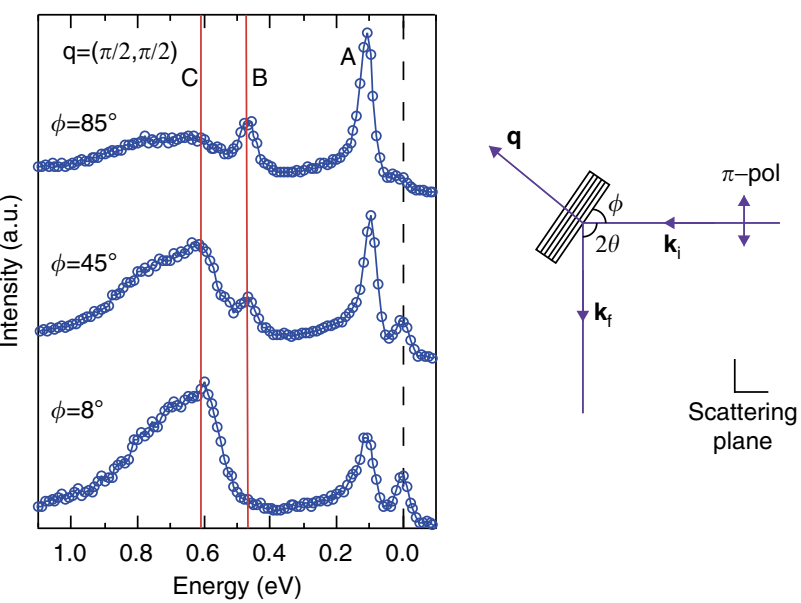

Figure $\mathbf{2}$ | RIXS spectra of $\mathbf{S r}_{\mathbf{2}} \mathbf{I r O}_{\mathbf{4}}$. (a) The spin-orbital level scheme of the three Kramers pairs and their orbital shapes in $\mathrm{Sr}_{2} \mathrm{IrO}_{4}$. The three-fold degenerate orbital states of $t_{2 \mathrm{~g}}$ symmetry under the cubic crystal field splits into a $j=1 / 2$ doublet and a $j=3 / 2$ quartet in the presence of spin-orbit coupling. The spin-orbit entangled nature of these quantum states is illustrated with colours; orange (blue) represent spin up (down) projection. A tetragonal crystal field further splits the $j=3 / 2$ quartet into two Kramers doublets labelled as B and C; B (C) represents states with $j_{z}$ quantum numbers $\pm 3 / 2( \pm 1 / 2)$. Spin-orbit exciton refers to an excitation of a hole from the $j=1 / 2$ manifold to the $j=3 / 2$ manifold. (b) Spectrum at $\mathbf{q}=(\pi / 2, \pi / 2)$ measured at three different $X$-ray incident angle $\phi$ of $8^{\circ}$, $45^{\circ}$ and $82^{\circ}$, corresponding to three-dimensional $\mathbf{q}$ of $(-3.5,3,25.5)$, $(0.5,0,34)$ and $(3.5,3,25.5)$, respectively, in reciprocal lattice units. A, B and $C$ denote the energy positions of the three Kramers pairs illustrated in $\mathbf{a}$. The scattering angle $2 \theta$ was kept within $5^{\circ}$ from $90^{\circ}$. 
a

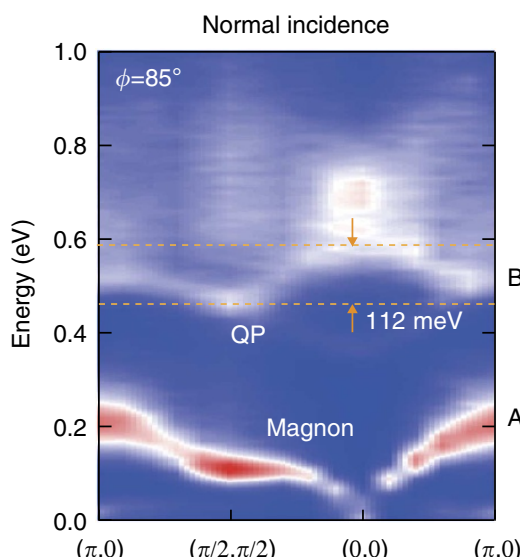

RIXS

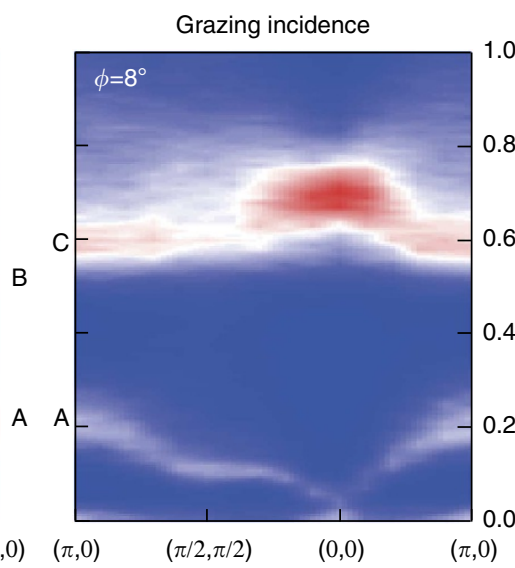

b

SCBA calculation
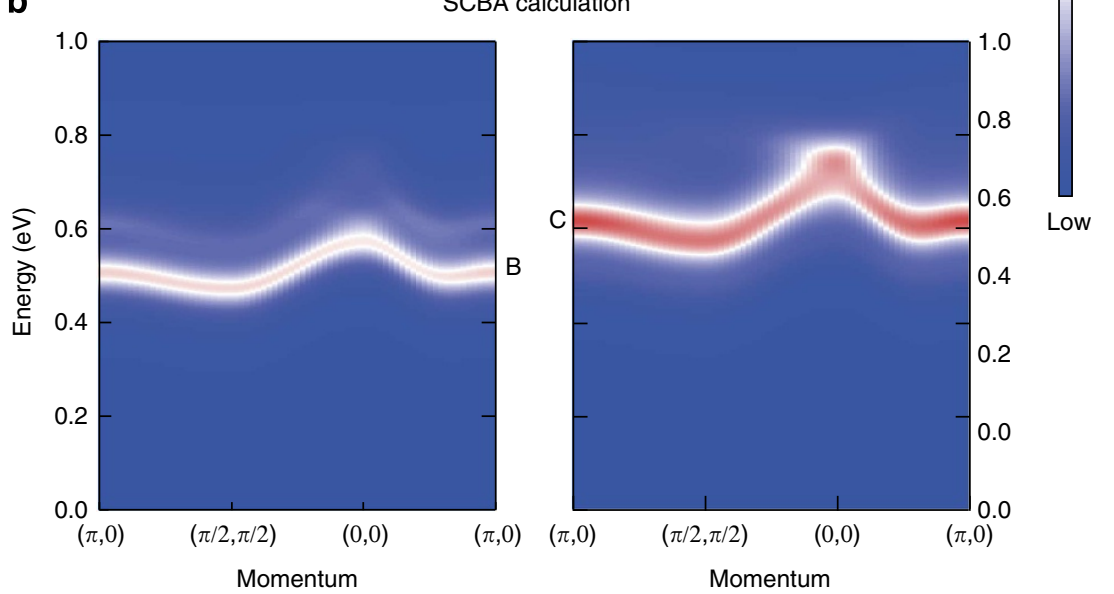

Figure 3 | Selective mapping of the two-exciton modes and their comparison to SCBA calculations. (a) Image plot of RIXS spectra measured along high-symmetry lines in the normal and grazing incidence geometry. (b) SCBA calculations using the parameters $\lambda_{\mathrm{SO}}=382 \mathrm{meV}, \Delta_{\mathrm{BC}}=137 \mathrm{meV}$, $t_{1}=J_{1} / 2=30 \mathrm{meV}, t_{2}=t_{3}=7.6 \mathrm{meV}, J_{1}=60 \mathrm{meV}, J_{2}=-20 \mathrm{meV}$ and $J_{3}=15 \mathrm{meV} . t_{1,2,3}\left(J_{1,2,3}\right)$ denote first, second and third nearest neighbour hoppings (magnetic couplings). $\lambda_{\mathrm{so}}$ and $\Delta_{\mathrm{BC}}$ are defined in Fig. 2 . The spectral functions obtained by SCBA calculations are convoluted with a Lorentzian function with $5 \mathrm{meV}$ width.

\section{Results}

Strong modulation of RIXS intensities as a function of incident $\mathrm{X}$-ray angle. Figure $2 \mathrm{~b}$ shows the RIXS spectra measured at three different incident angles $\phi$ of X-ray while fixing momentum transfer at $\mathbf{q}=(\pi / 2, \pi / 2)$. The two-exciton modes, labelled as peaks $\mathrm{B}$ and $\mathrm{C}$, show strong modulations in intensity as a function of $\phi$ through the change in the incident and outgoing X-ray polarizations relative to the sample surface and thereby the RIXS matrix elements. In particular, the peak B is strongly enhanced (completely suppressed) by tuning $\phi$ to normal (grazing) incidence geometry. This strong matrix element effect enables selective mapping of $\mathrm{B}$ and $\mathrm{C}$ modes, as shown in Fig. 3a. While both $\mathrm{B}$ and $\mathrm{C}$ modes display rather similar dispersions, the B mode has a much narrower linewidth (see Fig. 4c). We note that the electron-hole continuum having a threshold of $\approx 0.41 \mathrm{eV}$ (ref. 25 ) may strongly damp the $\mathrm{C}$ mode. With the twoexciton modes disentangled, our high-resolution measurement yields for the $\mathrm{B}$ mode a bandwidth of $\approx 112 \mathrm{meV}$. This finding is consistent with the expectation that the bandwidth is on the scale of a few times the antiferromagnetic exchange coupling $J$ of about $60 \mathrm{meV}$ (ref. 16). The global topology of the dispersions with minimum at $\mathbf{q}=(\pi / 2, \pi / 2)$ and maximum at the $\Gamma$ point precisely matches that measured for a hole in cuprates by ARPES ${ }^{9}$, which strongly supports the hole-versus-exciton analogy.
Justification of hole-versus-excition analogy. We compare the experimental data to the spectral function of the effective $t-J$ model calculated within the SCBA (Fig. $3 \mathrm{~b}$ and Supplementary Fig. 1). For details, see Supplementary Note 1 and ref. 16. The calculation yields two modes, one of which is predominantly from the Kramers doublet with quantum numbers $\mid j=3 / 2, j_{z}= \pm 3$ / $2>$, while the other has mostly $\mid j=3 / 2, j_{z}= \pm 1 / 2>$ character. Through an explicit calculation of the RIXS matrix elements, we find that their dependences on $\phi$ are such that the $j_{z}= \pm 1 / 2$ $\left(j_{z}= \pm 3 / 2\right)$ states, with more in-plane components, are enhanced in intensity in the grazing (normal) incidence geometry. This implies a level scheme of $j_{z}= \pm 1 / 2$ lying higher in energy (in the hole picture) than the $j_{z}= \pm 3 / 2$ states, which is opposite to expectations based on perfectly cubic or $c$ axis-elongated oxygen octahedra, but agrees with the results from non-resonant inelastic $\mathrm{X}$-ray scattering ${ }^{26}$, electron spin resonance ${ }^{27}$ and quantum chemistry calculations ${ }^{28}$. Taking a crystal field splitting $\Delta_{\mathrm{BC}}$ of $137 \mathrm{meV}$ and fixing all other parameters to values inferred from independent studies, the effective $t-J$ model reproduces the gross features of the experimental spectra. Details are improved by including further neighbouring hoppings, which are expected to be significant based on sizable further neighbour magnetic couplings ${ }^{16}$. With the parameters given in Fig. $3 \mathrm{~b}$ caption, we find an excellent agreement with the data in terms of the 

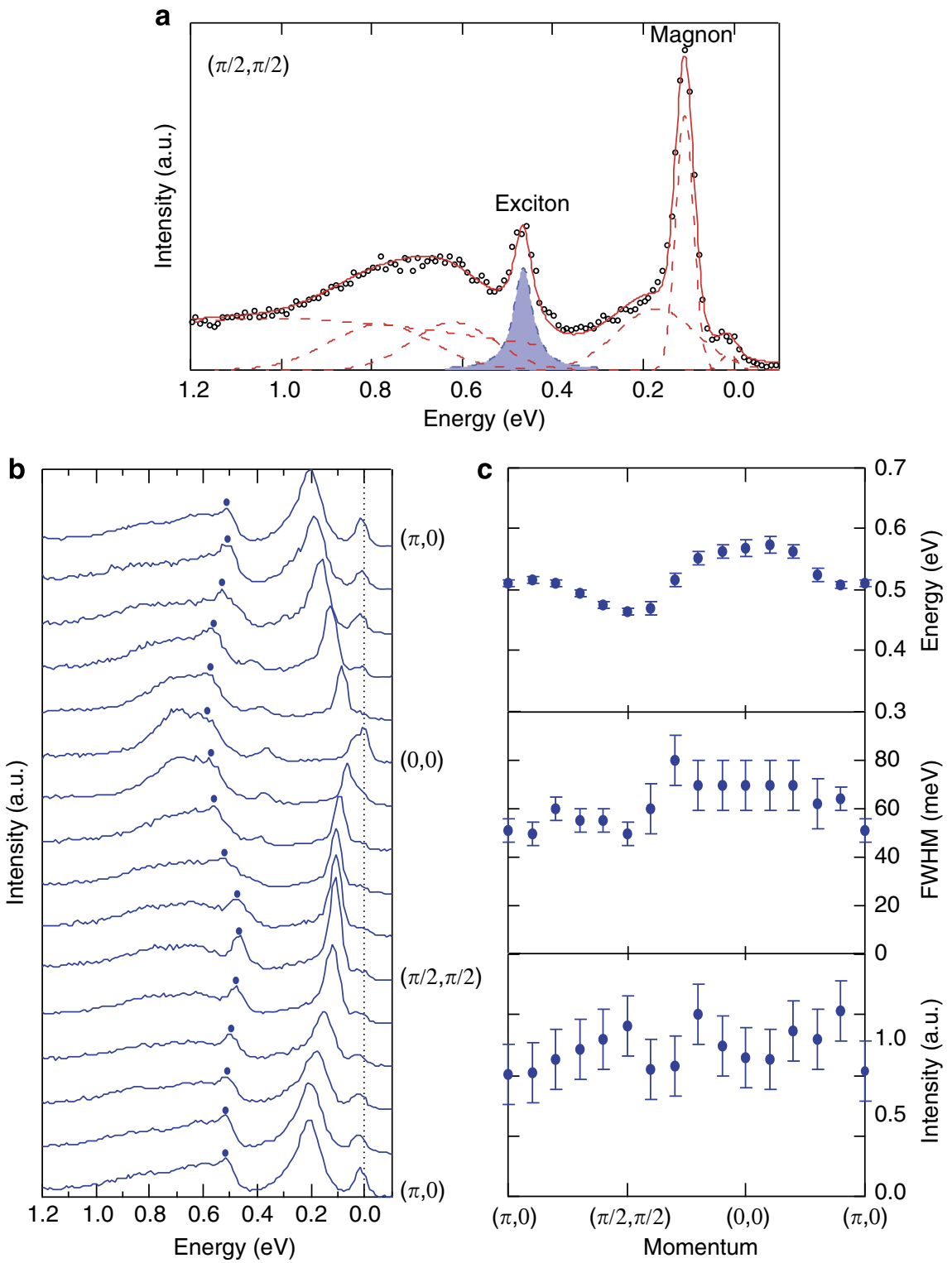

Figure 4 | Exciton dynamics in $\mathbf{S r}_{2} I_{\mathbf{r O}} \mathbf{O}_{\mathbf{4}}$. (a) RIXS spectrum at $\mathbf{q}=(\pi / 2, \pi / 2)$ (black open circles). The blue shaded peak corresponds to the exciton QP peak. The spectrum was fitted (red solid curve) using a Lorenzian lineshape for the exciton QP peak and Gaussian lineshapes for all other peaks (red dashed lines). The low-energy features relative to exciton QP consist of elastic, single- and double-magnon peaks, and high-energy features the sum of $\mathrm{C}$ mode and background due to electron-hole continuum and the incoherent part of B mode. (b) Stack plot of the image plot in Fig. 2a, left panel. Oval symbols mark the energy position of the $\mathrm{QP}$. In addition to the $\mathrm{QP}$, a small peak with the dispersion minimum at the $\Gamma$ point at $E \approx 0.37 \mathrm{eV}$ is observed. A similar peak has been observed in a related material $\mathrm{Na}_{2} \mid \mathrm{rO}_{3}$ and attributed to a bound state at the edge of the particle-hole continuum ${ }^{29}$.

(c) Energy, width and intensity of the QP peaks along high-symmetry lines extracted from peak fitting as exemplified in a. Error bars represent the s.d. in the data fitting procedure.

polarization dependence and the dispersion relations, confirming that the exciton dynamics is essentially captured by the effective $t-J$ model.

A well-defined excitonic $Q P$ in a $2 D$ spin-1/2 quantum AF. Having justified the hole-versus-exciton analogy, we now bring to light the key observation from the exciton spectra. The energy distribution curves, measured in the normal incidence geometry to highlight the $\mathrm{B}$ mode, reveal a very sharp exciton peak, most prominent at $\mathbf{q}=(\pi / 2, \pi / 2)$ (Fig. 4a) and resolved throughout most part of the Brillouin zone (Fig. $4 \mathrm{~b}$ ). We use a phenomenological Lorentzian lineshape to fit the spectra to extract the peak energy, width and intensity, which are summarized in Fig. 4c.
We note that the peak width is as narrow as $\approx 50 \mathrm{meV}$ (of which $40 \mathrm{meV}$ is intrinsic after deconvoluting the instrumental broadening) at $\mathbf{q}=(\pi / 2, \pi / 2)$. While such a sharp peak is not uncommon in RIXS spectra of iridates ${ }^{29}$, it is certainly much narrower than that of the sharpest peak $(\sim 200 \mathrm{meV})$ in the hole spectral function measured by ARPES for the same material ${ }^{13,30}$. The peak width is also much smaller than its total bandwidth $(\approx 112 \mathrm{meV}$ ), which establishes the exciton as a propagating mode in a solid, or a QP. More importantly, our observation of an excitonic QP establishes hard evidence that a particle can propagate coherently through a $2 \mathrm{D}$ spin-1/2 quantum $\mathrm{AF}$. Although dispersive excitons have been observed in classical three-dimensional magnets ${ }^{31-33}$, we are not aware of any excitonic QP observed in a $2 \mathrm{D}$ spin-1/2 quantum AF. This 
a

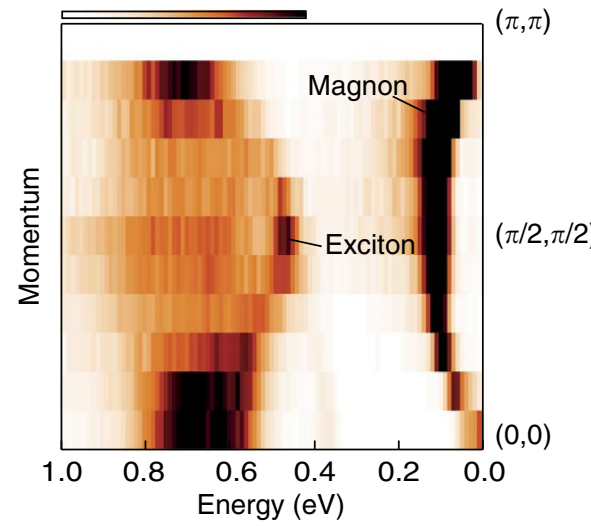

C

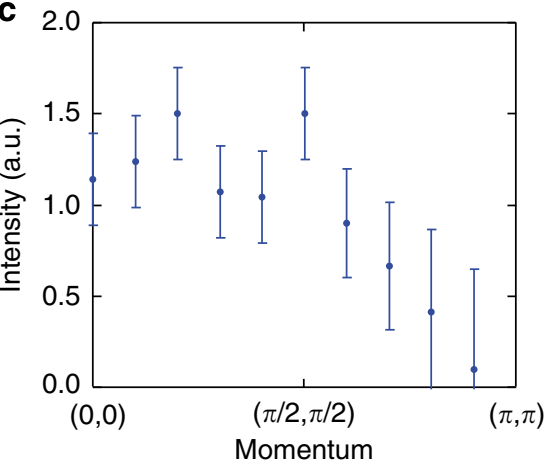

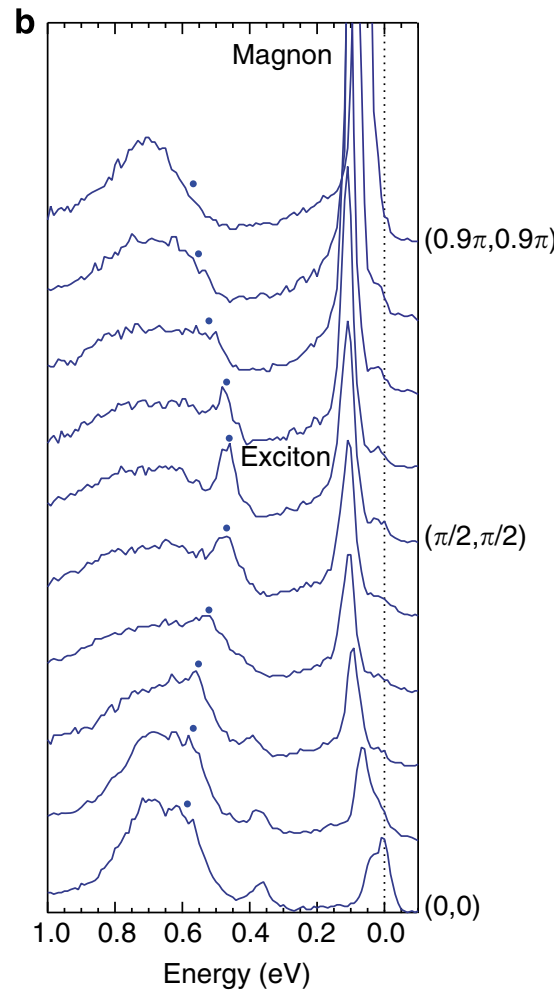

Figure 5 | Asymmetric exciton QP intensity. (a) Image plot of RIXS spectra along $(0,0)-(\pi, \pi)$ direction. (b) Stack plot of the image plot in a. Oval symbols mark the energy position of the QP. (c) Intensity of the QP peaks along $(0,0)-(\pi, \pi)$ direction. Error bars represent the s.d. in the data fitting procedure.

raises a fundamental question: why is a QP absent for the singlehole excitation?

\section{Discussion}

Thus far we have focused on the similarity between the dynamics of a hole and an exciton. Let us now discuss some important differences. A hole is a charge monopole and its sudden creation in the ARPES process leads to deformation of the surrounding ionic oxygen cage, which results in a strong hole-lattice coupling detrimental for the hole propagation. Further, a hole interacts with charged impurities always present and poorly screened in an insulator. Both of these effects have been shown to strongly damp or wash out sharp $\mathrm{QPs}^{34-36}$, thereby significantly redistributing the hole spectral function. In fact, most likely for these reasons, for a quasi-one-dimensional system $\mathrm{Sr}_{2} \mathrm{CuO}_{3}$ in which the phenomenon of spin-charge/orbital separation is established $^{37,38}$, ARPES measures much-broadened spectra ${ }^{39,40}$ of the theoretically predicted sharp edge-singularity in the exactly solvable model. By contrast, a charge-neutral exciton with a quadrupole moment should couple much more weakly to the lattice and is not subject to long-range Coulomb forces due to impurities. Thus, the exciton avoids these 'side effects' and reveals the intrinsic dynamics of the $t-J$ model that have remained elusive for the past several decades.

Our central message is that the unprecedentedly narrow linewidth of the QP allows a direct access into the nature of a QP living in the background of a $2 \mathrm{D}$ quantum spin-1/2 Heisenberg $\mathrm{AF}$, and thus that it allows direct verification of a class of theories that predict a finite QP residue in the Mott insulating phase, which has thus far remained inconclusive in the apparent absence of a QP in the hole channel. For instance, the observed exciton intensity can be compared with that calculated in theoretical models on a quantitative level. Figure $5 \mathrm{a}, \mathrm{b}$ shows the exciton dispersion along $(0,0)-(\pi, \pi)$ direction. The dispersion is symmetric with respect to $(\pi / 2, \pi / 2)$ point on the magnetic zone boundary, but the intensities inside and outside of the magnetic zone differ significantly (Fig. 5c); the exciton QP displays a steep drop in intensity as $(\pi / 2, \pi / 2)$ is crossed. This is a generic feature predicted for a hole in a $t-J$ model within an SCBA approach ${ }^{41}$ and indirectly inferred from ARPES measurements on cuprates ${ }^{42}$. Although some deviations from the cuprate physics might have been expected on general grounds due to fundamentally different nature of a hole and an exciton, and material-specific differences between $\mathrm{Sr}_{2} \mathrm{IrO}_{4}$ and cuprates, the striking agreement down to a level of fine details with theories constructed for hole dynamics in cuprates demonstrates excellent parallel between the low-energy physics of $\mathrm{Sr}_{2} \mathrm{IrO}_{4}$ and the cuprates.

\section{Methods}

RIXS measurements. A single crystal of $\mathrm{Sr}_{2} \mathrm{IrO}_{4}$ of $1 \mathrm{~mm} \times 1 \mathrm{~mm}$ size grown by the flux method was mounted in a displex closed-cycle cryostat and measured at $15 \mathrm{~K}$. The RIXS measurements were performed using the MERIX spectrometer at the 30 -ID beamline ${ }^{43}$ of the Advanced Photon Source. X-rays were

monochromatized to a bandwidth of $15 \mathrm{meV}$, and focused to have a beam size of $45(\mathrm{H}) \times 30(\mathrm{~V}) \mu \mathrm{m}^{2}$. A horizontal scattering geometry was used with the incident photon polarization in the scattering plane. A Si (844) diced spherical analyser with 4 inch radius and a position-sensitive silicon microstrip detector were used in the Rowland geometry. The overall energy resolution of the MERIX spectrometer at the $\mathrm{Ir} \mathrm{L}_{3}$ edge was $30 \mathrm{meV}$, as determined from the full-width-half-maximum of the elastic peak. The RIXS spectra were reproduced at least twice to check for consistency.

\section{References}

1. Lee, P. A., Nagaosa, N. \& Wen, X.-G. Doping a Mott insulator: Physics of high-temperature superconductivity. Rev. Mod. Phys. 78, 17-85 (2001).

2. Schmitt-Rink, S., Varma, C. M. \& Ruckenstein, A. E. Spectral function of holes in a quantum antiferromagnet. Phys. Rev. Lett. 60, 2793 (1988).

3. Kane, C. L., Lee, P. A. \& Read, N. Motion of a single hole in a quantum antiferromagnet. Phys. Rev. B 39, 6880 (1989). 
4. Duffy, D. et al. Hole-doping evolution of the quasiparticle band in models of strongly correlated electrons for the high- $T_{c}$ cuprates. Phys. Rev. B 56, 5597 (1997).

5. Eder, R., Ohta, Y. \& Sawatzky, G. A. Doping-dependent quasiparticle band structure in cuprate superconductors. Phys. Rev. B 55, R3414 (1996).

6. Anderson, P. W. 'Luttinger-liquid' behavior of the normal metallic state of the 2D Hubbard model. Phys. Rev. Lett. 64, 1839 (1990).

7. Laughlin, R. B. Evidence for quasiparticle decay in photoemission from underdoped cuprates. Phys. Rev. Lett. 79, 1726 (1997).

8. Sheng, D. N., Chen, Y. C. \& Weng, Z. Y. Phase string effect in a doped antiferromagnet. Phys. Rev. Lett. 77, 5102 (1996).

9. Wells, B. O. et al. E versus $k$ relations and many body effects in the model insulating copper oxide $\mathrm{Sr}_{2} \mathrm{CuO}_{2} \mathrm{Cl}_{2}$. Phys. Rev. Lett. 74, 964-967 (1995).

10. Tohyama, T. \& Maekawa, S. Angle-resolved photoemission in high Tc cuprates from theoretical viewpoints. Supercond. Sci. Technol. 13, R17-R32 (2000).

11. Shen, K. M. et al. Missing quasiparticles and the chemical potential puzzle in the doping evolution of the cuprate superconductors. Phys. Rev. Lett. 93, 267002 (2004)

12. Ye, C. et al. Visualizing the atomic-scale electronic structure of the $\mathrm{Ca}_{2} \mathrm{CuO}_{2} \mathrm{Cl}_{2}$ Mott insulator. Nat. Commun. 4, 1365 (2013).

13. Kim, B. J. et al. Novel $J_{\text {eff }}=1 / 2$ Mott state induced by relativistic spin-orbit coupling in $\mathrm{Sr}_{2} \mathrm{IrO}_{4}$. Phys. Rev. Lett. 101, 076402 (2008).

14. Jackeli, G. \& Khaliullin, G. Mott insulators in the strong spin-orbit coupling limit: from Heisenberg to a quantum compass and Kitaev models. Phys. Rev. Lett. 102, 017205 (2009).

15. Kim, B. J. et al. Phase-sensitive observation of a spin-orbital Mott state in $\mathrm{Sr}_{2} \mathrm{IrO}_{4}$. Science 323, 1329-1332 (2009).

16. Kim, J. et al. Magnetic excitation spectra of $\mathrm{Sr}_{2} \mathrm{IrO}_{4}$ probed by resonant inelastic $\mathrm{X}$-ray scattering: establishing links to cuprate superconductors. Phys. Rev. Lett. 108, 177003 (2012).

17. Fujiyama, S. et al. Two-dimensional Heisenberg behavior of $J_{\text {eff }}=1 / 2$ isospins in the paramagnetic state of the spin-orbital Mott insulator $\mathrm{Sr}_{2} \mathrm{IrO}_{4}$. Phys. Rev. Lett. 108, 247212 (2012).

18. Crawford, M. K. et al. Structural and magnetic studies of $\mathrm{Sr}_{2} \mathrm{IrO}_{4}$. Phys. Rev. B 49, 9198-9201 (1994)

19. Wang, F. \& Senthil, T. Twisted Hubbard model for $\mathrm{Sr}_{2} \mathrm{IrO}_{4}$ : Magnetism and possible high temperature superconductivity. Phys. Rev. Lett. 106, 136402 (2011).

20. Martins, C., Aichhorn, M., Vaugier, L. \& Biermann, S. Reduced effective spin-orbital degeneracy and spin-orbital ordering in paramagnetic transitionmetal oxides: $\mathrm{Sr}_{2} \mathrm{IrO}_{4}$ versus $\mathrm{Sr}_{2} \mathrm{RhO}_{4}$. Phys. Rev. Lett. 107, 266404 (2011).

21. Arita, R., Kuneš, J., Kozhevnikov, A. V., Eguiluz, A. G. \& Imada, M. Ab initio studies on the interplay between spin-orbit interaction and coulomb correlation in $\mathrm{Sr}_{2} \mathrm{IrO}_{4}$ and $\mathrm{Ba}_{2} \mathrm{IrO}_{4}$. Phys. Rev. Lett. 108, 086403 (2012).

22. Hsieh, D., Mahmood, F., Torchinsky, D. H., Cao, G. \& Gedik, N. Observation of a metal-to-insulator transition with both Mott-Hubbard and Slater characteristics in $\mathrm{Sr}_{2} \mathrm{IrO}_{4}$ from time-resolved photocarrier dynamics. Phys. Rev. $B$ 86, 035128 (2012).

23. Li, Q. et al. Microscopic and spectroscopic evidence for a Slater metal-insulator transition in $\mathrm{Sr}_{2} \mathrm{IrO}_{4}$. Preprint at http://arxiv.org/abs/1303.7265.

24. Yamasaki, A. et al. Bulk nature of layered perovskite iridates beyond the Mott scenario: An approach from bulk sensitive photoemission study. Phys. Rev. B 89, 121111(R) (2014).

25. Moon, S. J. et al. Temperature dependence of the electronic structure of the $J_{\text {eff }}=12$ Mott insulator $\mathrm{Sr}_{2} \mathrm{IrO}_{4}$ studied by optical spectroscopy. Phys. Rev. B 80, 195110 (2009).

26. Fujiyama, S. et al. Spin and orbital contributions to magnetically ordered moments in $5 d$ layered perovskite $\mathrm{Sr}_{2} \mathrm{IrO}_{4}$. Phys. Rev. Lett. 112, 016405 (2014).

27. Bahr, S. et al. Low energy magnetic excitations in the spin-orbital Mott insulator $\mathrm{Sr}_{2} \mathrm{IrO}_{4}$. Phys. Rev. B 89, 180401(R) (2014).

28. Katukuri, V. M., Stoll, H., van den Brink, J. \& Hozoi, L. Ab initio determination of excitation energies and magnetic couplings in correlated quasi-twodimensional iridates. Phys. Rev. B 85, 220402(R) (2012).
29. Gretarsson, H. et al. Crystal-field splitting and correlation effect on the electronic structure of $\mathrm{A}_{2} \mathrm{IrO}_{3}$. Phys. Rev. Lett. 110, 076402 (2013).

30. Wang, Q. et al. Dimensionality-controlled Mott transition and correlation effects in single-layer and bilayer perovskite iridates. Phys. Rev. B 87, 245109 (2013).

31. Holden, T. M. et al. Excitations in KCoF3. J. Phys. C 4, 2127 (1971).

32. Buyers, W. J. L., Holden, T. M., Svensson, E. C., Cowley, R. A. \& Hutchings, M. T. Excitations in KCoF3. J. Phys. C 4, 2139 (1971).

33. Jensen, J. \& Mackintosh, A. R. Rare Earth Magnetism (Clarendon Press, 1991).

34. Mishchenko, A. S. \& Nagaosa, N. Electron-phonon coupling and a polaron in thet-J model: from weak to the strong coupling regime. Phys. Rev. Lett. 93, 036402 (2004).

35. Chen, W., Khaliullin, G. \& Sushkov, O. P. Coulomb disorder effects on angleresolved photoemission and nuclear quadrupole resonance spectra in cuprates. Phys. Rev. B 80, 094519 (2009).

36. King, P. D. C. et al. Spectroscopic evidence for polaronic behaviour of the strong spin-orbit insulator $\mathrm{Sr}_{3} \mathrm{Ir}_{2} \mathrm{O}_{7}$. Phys. Rev. B 87, 241106(R) (2013).

37. Zaliznyak, I. A. et al. Spinons in the strongly correlated copper oxide chains in $\mathrm{SrCuO}_{2}$. Phys. Rev. Lett. 93, 087202 (2004).

38. Schlappa, J. et al. Spin-orbital separation in the quasi-one-dimensional Mott insulator $\mathrm{Sr}_{2} \mathrm{CuO}_{3}$. Nature 485, 82-85 (2011).

39. Kim, C. et al. Observation of spin-charge separation in one-dimensional $\mathrm{SrCuO}_{2}$. Phys. Rev. Lett. 77, 4054-4057 (1996).

40. Kim, B. J. et al. Distinct spinon and holon dispersions in photoemission spectral functions from one-dimensional $\mathrm{SrCuO}_{2}$. Nat. Phys. 2, 397-401 (2006).

41. Sushkov, O. P., Sawatzky, G. A., Eder, R. \& Eskes, H. Hole photoproduction in insulating copper oxide. Phys. Rev. B 56, 11769 (1997).

42. LaRosa, S. et al. Electronic structure of $\mathrm{CuO}_{2}$ planes: From insulator to superconductor. Phys. Rev. B 56, R525 (1997).

43. Gog, T. et al. Spherical analyzers and monochromators for resonant inelastic hard X-ray scattering: a compilation of crystals and reflections. J. Synchrotron Rad. 20, 74-79 (2013).

\section{Acknowledgements}

B.J.K. thanks G. Jackeli for discussions. Work in the Materials Science Division of Argonne National Laboratory (sample preparation and characterization) was supported by the US Department of Energy (DOE) Office of Science, Basic Energy Sciences, Materials Science and Engineering Division. Use of the Advanced Photon Source, an Office of Science User Facility operated for the US DOE Office of Science by Argonne National Laboratory, was supported by the US DOE under Contract No. DE-AC02-06CH11357.

\section{Author contributions}

J.K. and B.J.K. conceived and performed the experiment. T.G. contributed to the experiment. A.H.S. developed the analyser. B.J.K. prepared the sample. M.D. performed the numerical calculations; M.D., J.vdB., and G.K. developed the theoretical model. All authors discussed the results. B.J.K. led the manuscript preparation with contributions from all authors.

\section{Additional information}

Supplementary Information accompanies this paper at http://www.nature.com/ naturecommunications

Competing financial interests: The authors declare no competing financial interests

Reprints and permission information is available online at http://npg.nature.com/ reprintsandpermissions/

How to cite this article: Kim, J. et al. Excitonic quasiparticles in a spin-orbit Mott insulator. Nat. Commun. 5:4453 doi: 10.1038/ncomms5453 (2014). 